\title{
A STRETCHABLE AND TRANSPARENT SWNT STRAIN SENSOR ENCAPSULATED IN THIN PDMS FILMS
}

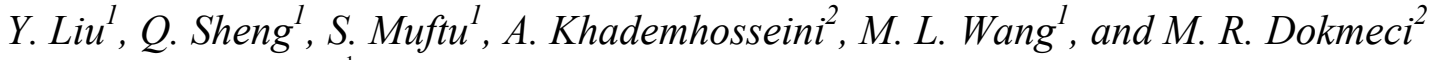 \\ ${ }^{1}$ Northeastern University, Boston, MA, USA \\ ${ }^{2}$ Brigham and Women's Hospital, Harvard Medical School, Boston, MA, USA
}

\begin{abstract}
In this paper, a stretchable and transparent strain sensor made of an ultrathin film of single-walled carbon nanotubes (SWNTs) encapsulated inside poly(dimethyl siloxane) (PDMS) films is presented. A surface plasma treatment, followed by a solution based, low temperature drop casting process was developed and utilized to fabricate the strain sensor. The fabricated SWNTs strain sensor functions as a two-terminal resistor which changes its resistance when the sensor is stretched. The strain sensor was tested with $10 \%$ tensile cyclic strain and was found to handle strains of up to $35 \%$. With PDMS encapsulation, the effect of environmental gases to the response of the SWNT strain sensors was eliminated. The electrical response of the SWNT strain sensor was highly reversible and repeatable after the initial conditioning step. The reliability of this sensor was evaluated up to 200 cycles at $4 \%$ strain. Our results demonstrate the possibility of using ultrathin SWNT films as a stretchable, transparent and reliable strain sensor.
\end{abstract}

\section{KEYWORDS}

Strain sensor, stretchable, transparent, flexible, SWNTs, PDMS, thin films

\section{INTRODUCTION}

Strain sensors previously fabricated on stainless steel or silicon substrates had flexibility yet lacked stretchability. The measurement range was normally less than $2 \%$ strain due to the limited ductility of traditional piezoresistive materials $[1,2]$. Stretchable devices while preserving the functionalities of the traditional strain sensors can at the same time be bendable, twistable and stretchable. As a deformable and conformable platform, stretchable strain sensors have great potential in implantable diagnostics, such as sensing neural signals from the human brain [3] and detecting dynamic mechanical forces exerted on the heart tissue [4]. Another potential application of stretchable strain sensors is the input screen of flexible displays [5] which requires that the strain sensor is both bendable and transparent.

Recently, various stretchable devices were developed by transfer printing, which transfered the free-standing functional layers (normally on silicon) from a mother wafer to a prestrained polymer substrate to form wavy or buckled structures [6-8]. For instance, Choi et. al. transferred $100 \mathrm{~nm}$ thick silicon membranes onto a prestrained PDMS substrate. After releasing the prestrain, the silicon membrane formed a two-dimensional (2D) wavy structure spontaneously on the elastomeric substrate. They demonstrated that the resulting structure can undergo $10 \% \sim 20 \%$ strain which is 10 to 20 times of the fracture limit of silicon [6]. Kim et. al. created stretchable electronics by using buckled, arc-shaped silicon ribbons as the interconnects for arrays of transistors, CMOS inverters, ring oscillators, and differential amplifiers on a PDMS substrate [8]. The arcshaped silicon ribbons were formed by bonding silicon thin films on a prestrained PDMS substrate at the nodes. After releasing the prestrain, the compressive forces caused the connecting bridges (the ribbons between two nodes) to be lifted from the PDMS substrate, thereby forming arc-shaped structures. Also, Khang and coworkers transferred SWNTs individually aligned on quartz to a prestrained PDMS substrate. The SWNTs formed buckled structures after the prestrain was released. This device was used to measure strains of up to 4\% [7] However, transfer printing is a fairly sophisticated and low yield approach to fabricate stretchable devices. In addition, the stretchability of the devices was limited to $20 \%$ and the devices were not transparent.

Another approach to fabricate stretchable strain sensors is to use materials with inherent stretchability such as conductive polymers [9], thin metal films [10] and insulating polymer matrices with conductive dopants $[11$, 12] as the functional layers on elastomeric substrates. Carbon nanotubes (CNTs) exhibit exceptionally high carrier mobility, substantial mechanical strength and unique electromechanical properties. The electrical conductance of a SWNT is extremely sensitive, repeatable and hysteresis free with various deformation modes such as bending and stretching [13]. Thin films of SWNTs composed of randomly orientated, entangled network of nanotubes exhibit superior robustness in terms of stretching, bending and abrasion. Nanotube networks could maintain electrical conductivity for strains up to $700 \%$ [14]. Thus SWNT films are promising candidates for stretchable strain sensors.

In this paper, utilizing drop casting and surface treatment, we demonstrate a novel ultrathin SWNT film based stretchable and transparent strain sensor. The drop casting process can easily deposit thin films of SWNTs by dispensing a SWNT solution onto a wide variety of substrates with different patterns. Thus ultrathin films of SWNTs can be mass produced and cost-effectively scaled to large areas by printing techniques. Besides, the solution based deposition process is compatible with microfabrication of devices on polymer substrates for stretchable electronics. Since the electrical properties of SWNTs are known to be influenced by the environment, the SWNT sensor was encapsulated with another PDMS layer on top (Figure 1). The fabricated SWNT strain sensor was tested with tensile strain and the stress/ resistance of the sensor was monitored simultaneously. The effect of encapsulation and the reliability of the sensor were also evaluated. 


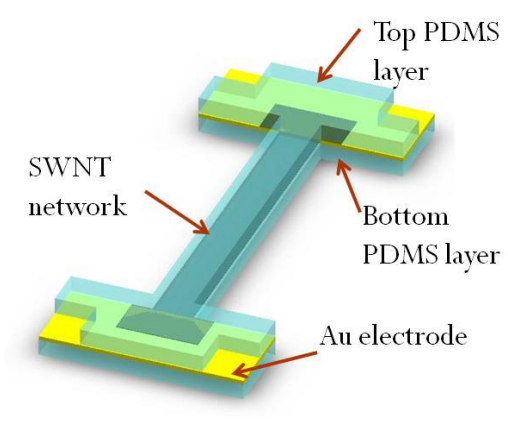

Figure 1: A schematic of the encapsulated SWNT strain sensor on a PDMS substrate.

\section{FABRICATION}

The fabrication process for the encapsulated SWNT strain sensors on a PDMS substrate is briefly illustrated in Figure 2. A 3" silicon wafer was first spun coated with a lift off resist (LOR 30B) to render the surface hydrophobic and to reduce the adhesion force between the PDMS thin film and the silicon substrate. This resist layer will facilitate easier removal of the device from the silicon wafer at the end of fabrication process. PDMS prepolymer was mixed with its curing agent with a ratio of $10: 1$ by weight (Sylgard 184 elastomer from Dow Corning). The mixed PDMS solution was degassed in a vacuum to remove bubbles and then spun coated on a silicon wafer (Figure 2a). The spin coated PDMS layer on a silicon wafer was cured in a $70{ }^{\circ} \mathrm{C}$ oven for 2 hours and the thickness of the PDMS film was measured to be $200 \mu \mathrm{m}$. $30 \mathrm{~nm} / 150 \mathrm{~nm}$ Cr/Au electrodes were selectively deposited on the PDMS substrate using a shadow mask made of $1 \mathrm{~mm}$ thick PDMS layer (Figure 2b). After peeling off the shadow mask, the PDMS substrate with $\mathrm{Au}$ electrodes were roughened by an oxygen plasma for 30 seconds using an inductively coupled plasma tool (Plasmatherm 790), as shown in Figure 2c. This step rendered the surface of the PDMS thin film hydrophilic and improved the spread of the SWNT solution onto the PDMS substrate. Commercial SWNTs (diameter: 1 2 nm and length: $2 \sim 5 \mu \mathrm{m}$ ) dispersed in DI water were sonicated for 2 hours to further debundle the SWNTs. The SWNT solution $(\sim 4 \mathrm{mg} / \mathrm{mL})$ was dispensed onto the roughened PDMS substrate (Figure 2d) and dried in air for 2 hours (Figure 2e). The dried SWNT network was held onto the polymer substrate by strong van der Waals forces [7, 15]. Then another PDMS layer with a thickness of $200 \mu \mathrm{m}$ was spun coated on top of the device to encapsulate the nanotube network and was cured in the $70{ }^{\circ} \mathrm{C}$ oven for 2 hours (Figure 2f). The PDMS layer residing above the $\mathrm{Au}$ microelectrodes and the excess PDMS on the silicon wafer were cut with a knife and were removed manually.

In this study, ten devices were fabricated and the average resistance of the strain sensors was $2.5 \pm 0.3 \mathrm{~K}$. The corresponding optical transparency is about $80 \%$. Then, the fabricated SWNT strain sensor was peeled off from the silicon wafer and was ready for mechanical testing. The structure and dimensions of the strain sensor is illustrated in Figure 1. The sensing area of the "dog bone" structure is the middle $(5 \mathrm{~mm} \times 40 \mathrm{~mm})$ rectangular area and the entire device thickness is around $400 \mu \mathrm{m}$. The "dog bone" design ensured that the normal strain was confined to the sensing area when stretched from the two

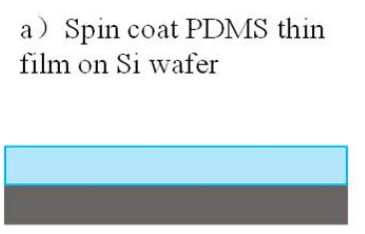

b) Deposit Au electrode

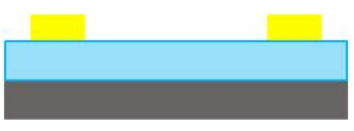

c) Roughen the surface with Plasma

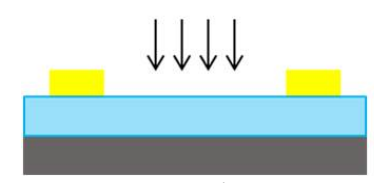

Figure 2: Fabrication process for the encapsulated SWNT strain sensor on a PDMS substrate.

ends of the sample. Before testing, the Au pads at the ends of the "dog bone" shaped sample were glued to $250 \mu \mathrm{m}$ thick cardboards to prevent slippage of the sample from the clamps. The SWNT thin film embedded in PDMS layers is transparent and flexible as shown in Figure 3a and a SEM micrograph of the drop casted SWNT network is shown in Figure 3b.

Tensile testing was conducted by a universal testing machine UMT-2 (CETR, Campbell, CA) in ambient environment. The change in resistance of the encapsulated SWNT sensor was monitored by a multimeter (HP 34401A) and displayed by a control panel designed by the LabVIEW program during testing. Meanwhile, the applied force and change in the length of the SWNT sensor were measured and recorded by a UMT-2 microtester. From the measured force and change in length, the normal stress and normal strain on the sample were calculated.

\section{RESULTS AND DISCUSSION}

In order to demonstrate strain sensing capability of the encapsulated thin SWNT layer, 10\% tensile strain was applied to the sensor at a rate of $0.02 \mathrm{~mm} / \mathrm{sec}$ while the resistance of the network was monitored simultaneously. The initial resistance of the SWNT sensor before testing was measured to be $2.83 \mathrm{~K} \Omega$. The stress-strain characteristics and the electrical response of SWNT

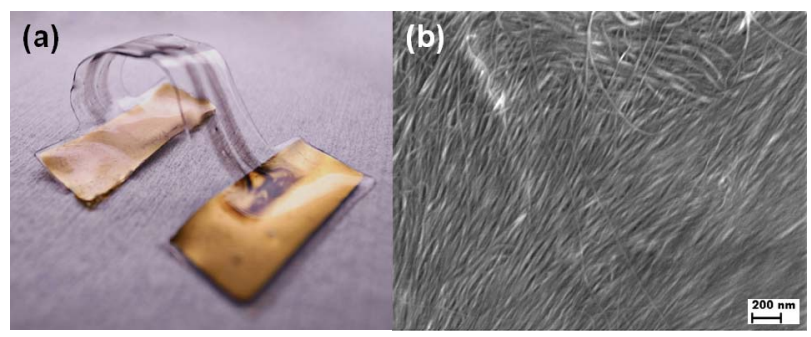

Figure 3: (a) Photograph of the fabricated transparent and flexible strain sensor. (b) SEM image of the SWNT network (scale bar is 200nm). 
sensor to $10 \%$ strain are shown in Figure $4 \mathrm{a}$ and $4 \mathrm{~b}$, respectively. The normal stress linearly increased with increasing normal strain up to $10 \%$. This indicates that $10 \%$ strain is in the linear elastic region of the PDMS substrate. However, the change in resistance $\left(\Delta R / R_{0}\right)$ of the SWNT network when loaded with $10 \%$ strain has two linear regions ( 0 to $3 \%$ and 6 to $10 \%$ ) with different slopes. The slope which represents the gauge factor (GF) of the SWNT strain sensor is calculated as follows,

$$
G F=\frac{\Delta R / R_{0}}{\mathcal{E}}
$$

From $1 \%$ to $3 \%$ strain, the slope of the SWNT strain sensor was about 0.36 , while from $6 \%$ to $10 \%$ strain, the slope was about 6.30 . The two linear regions in the electrical response of SWNTs to strain might indicate two different deformation regimes in the SWNT network. The SWNT strain sensor was shown to accommodate strains as large as 35\% indicated by an increase in its resistance with applied strain.

The conductance of a SWNT is sensitive to numerous gases in the environment, including moisture, methanol, etc. Therefore encapsulation of SWNTs is necessary to create a sensor which is not susceptible to changes in the environment. The effectiveness of the PDMS encapsulation was verified by a moisture test. In this study, saturated water vapor at $30^{\circ} \mathrm{C}$ was blown over the encapsulated and unencapsulated nanotube sensors for 20 seconds and then the devices were exposed to air at room temperature. As illustrated in Figure 5a, the change in resistance of the unencapsulated SWNT devices was
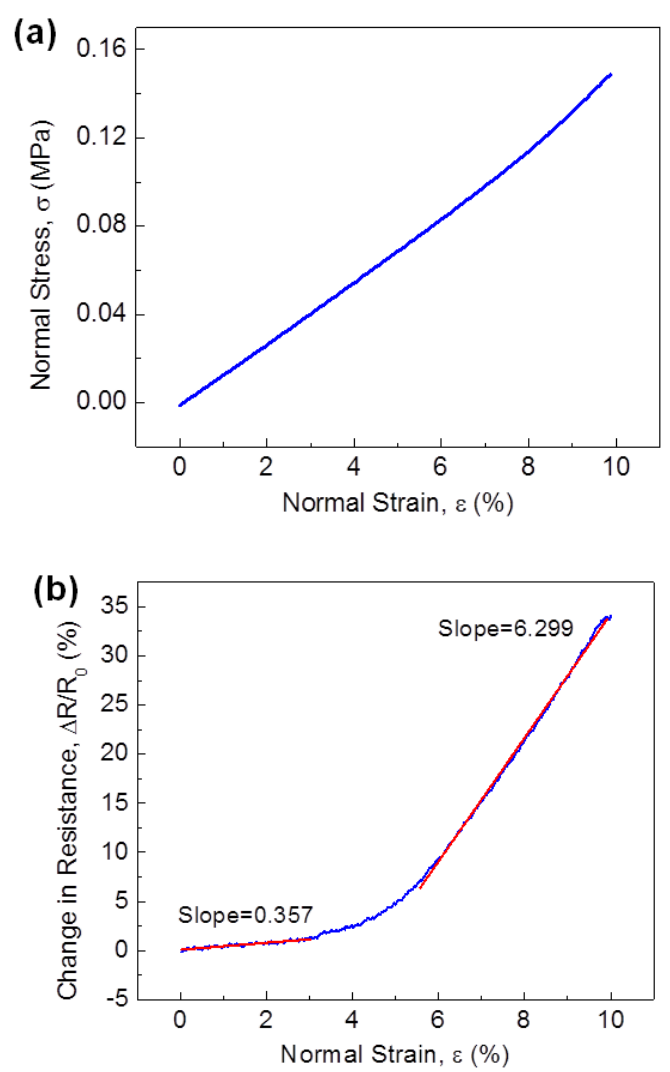

Figure 4: (a) The stress-strain characteristics of the SWNT sensor as the strain was increased from 0 to $10 \%$. (b) The change in resistance of the SWNTs as the sensors were exposed to 0 to $10 \%$ strain. about $20 \%$ when exposed to moisture, while the encapsulated SWNT strain sensors did not show any response to moisture. This result demonstrated that covering the SWNT network with a thin PDMS film can effectively eliminate the sensitivity of nanotubes to variations in humidity in the ambient environment. The changes in resistance of the encapsulated and the unencapsulated SWNT strain sensors versus strain are plotted in Figure 5b. The electro-mechanical behavior of the sealed nanotube sensor was very similar to that of the unsealed strain sensor. In this case, the electrical response of the unencapsulated SWNTs to strain is slightly higher than that of the encapsulated SWNTs which might be due to sample to sample variations.

Next, we tested the reliability of the SWNT strain sensor by performing cyclical loading for 200 cycles in the $0 \sim 4 \%$ strain range. The normal stress and the change in resistance as a function of normal strain for the $1^{\text {st }}, 50^{\text {th }}$, $100^{\text {th }}, 150^{\text {th }}$ and $200^{\text {th }}$ loading cycle are compared in Figure 6. Despite a slightly larger electrical response to strain in the first loading cycle, the changes in resistance of the $50^{\text {th }}, 100^{\text {th }}, 150^{\text {th }}$ and $200^{\text {th }}$ loading cycles are very close and hysteresis-free. The normal stress during 200 measurement cycles was perfectly linear with the normal strain. Our results indicated that the SWNT thin films embedded in PDMS thin films maintained their properties during extended testing cycles.
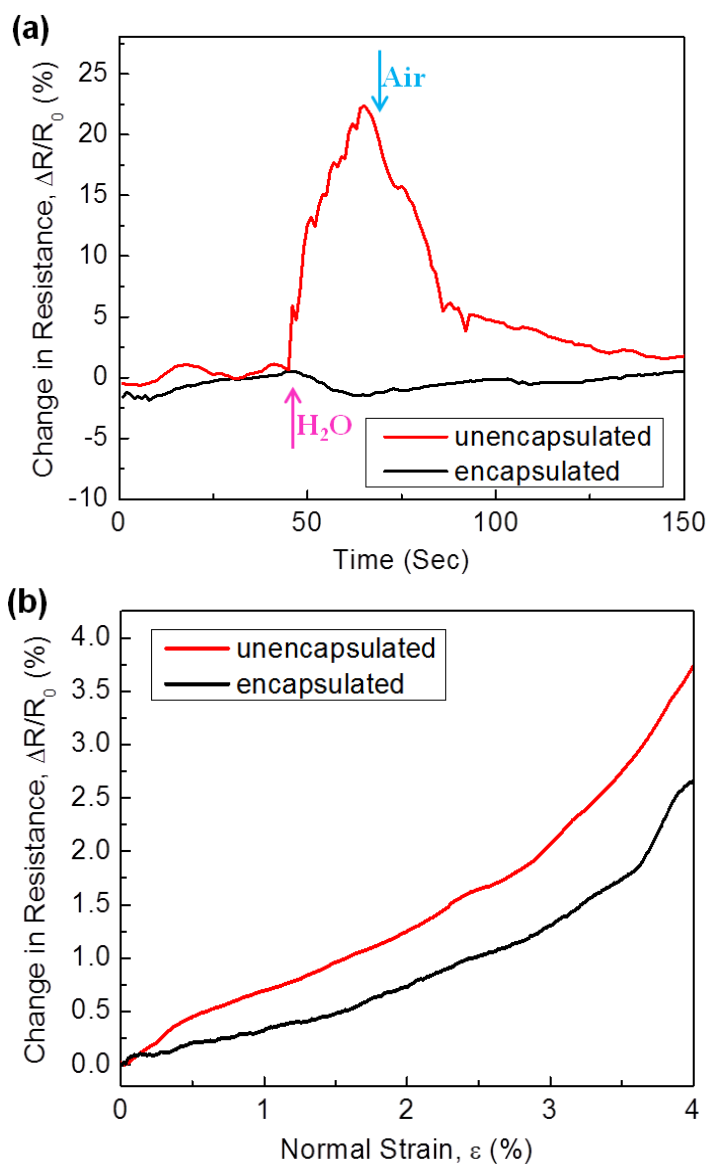

Figure 5: The change in resistance of the encapsulated and unencapsulated SWNT strain sensor when exposed to saturated moisture $(a)$, when under strain $(b)$. 


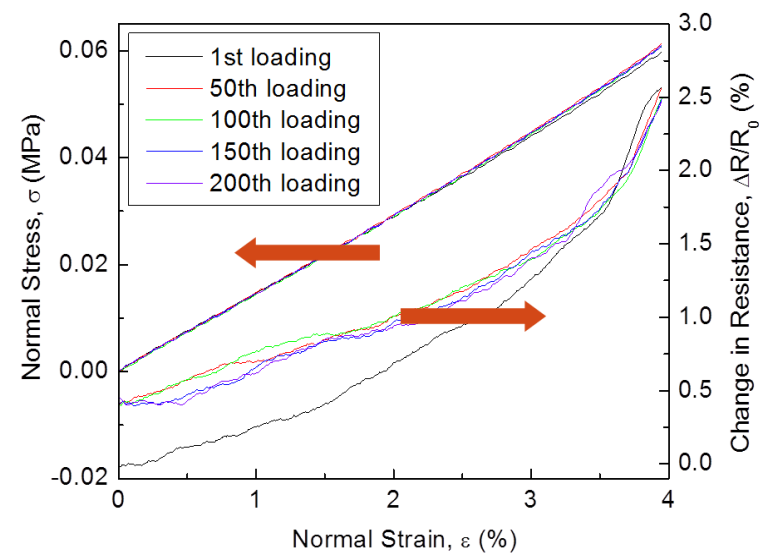

Figure 6: Normal stress and the change in resistance versus strain for 200 cycles.

\section{CONCLUSIONS}

We developed a stretchable and transparent strain sensor composed of SWNT thin films encapsulated in PDMS films. The SWNT strain sensor can measure strains of up to $35 \%$. The response of the SWNT strain sensor to $4 \%$ strain was highly repeatable and reversible. The changes in the sensor response due to variations in the environment were eliminated by encapsulation with a PDMS film. The SWNT network in PDMS thin films as a flexible, stretchable and transparent strain sensor might find potential applications in implantable diagnostic devices, stretchable monitors and large area electronic displays.

\section{ACKNOWLEDGEMENTS}

This research was conducted at the George J. Kostas Nanoscale Technology and Manufacturing Research Center at Northeastern University. The authors would like to kindly acknowledge funding from NSF for Grants 0731102 and 0947874. The SWNTs utilized in this project was provided by Brewer Science Inc.

\section{REFERENCES}

[1] R. G. Azevedo, D. G. Jones, A. V. Jog, B. Jamshidi, D. R. Myers, L. Chen, X. A. Fu, M. Mehregany, M. B. J. Wijesundara, and A. P. Pisano, "A SiC MEMS resonant strain sensor for harsh environment applications," IEEE Sensors Journal, vol. 7, pp. 568576, Mar-Apr 2007.

[2] Y. Kim, Y. Kim, C. Lee, and S. Kwon, "Thin polysilicon gauge for strain measurement of structural elements," IEEE Sensors Journal, vol. 10, pp. 1320-1327, Aug 2010.

[3] Z. Yu, O. Graudejus, S. P. Lacour, S. Wagner, and B. Morrison, 3rd, "Neural sensing of electrical activity with stretchable microelectrode arrays," Conf Proc IEEE Eng Med Biol Soc, vol. 2009, pp. 4210-3, 2009.

[4] D. H. Kim, N. Lu, R. Ghaffari, Y. S. Kim, S. P. Lee, L. Xu, J. Wu, R. H. Kim, J. Song, Z. Liu, J. Viventi, B. de Graff, B. Elolampi, M. Mansour, M. J. Slepian, S. Hwang, J. D. Moss, S. M. Won, Y. Huang, B. Litt, and J. A. Rogers, "Materials for multifunctional balloon catheters with capabilities in cardiac electrophysiological mapping and ablation therapy,"
Nat Mater, vol. 10, pp. 316-23, Apr 2011.

[5] S. Takamatsu, T. Takahata, M. Muraki, E. Iwase, K. Matsumoto, and I. Shimoyama, "Transparent conductive-polymer strain sensors for touch input sheets of flexible displays," Journal of Micromechanics and Microengineering, vol. 20, Jul 2010.

[6] W. M. Choi, J. Z. Song, D. Y. Khang, H. Q. Jiang, Y. Y. Huang, and J. A. Rogers, "Biaxially stretchable "Wavy" silicon nanomembranes," Nano Letters, vol. 7, pp. 1655-1663, Jun 2007.

[7] D. Y. Khang, J. L. Xiao, C. Kocabas, S. MacLaren, T. Banks, H. Q. Jiang, Y. Y. G. Huang, and J. A. Rogers, "Molecular scale buckling mechanics on individual aligned single-wall carbon nanotubes on elastomeric substrates," Nano Letters, vol. 8, pp. 124130, Jan 2008.

[8] D. H. Kim, J. Z. Song, W. M. Choi, H. S. Kim, R. H. Kim, Z. J. Liu, Y. Y. Huang, K. C. Hwang, Y. W. Zhang, and J. A. Rogers, "Materials and noncoplanar mesh designs for integrated circuits with linear elastic responses to extreme mechanical deformations," Proceedings of the National Academy of Sciences of the United States of America, vol. 105, pp. 1867518680, Dec 2008.

[9] T. S. Hansen, K. West, O. Hassager, and N. B. Larsen, "Highly stretchable and conductive polymer material made from poly $(3,4-$ ethylenedioxythiophene) and polyurethane elastomers," Advanced Functional Materials, vol. 17, pp. 3069-3073, Nov 2007.

[10] J. Jones, S. P. Lacour, S. Wagner, and Z. G. Suo, "Stretchable wavy metal interconnects," Journal of Vacuum Science \& Technology A, vol. 22, pp. 17231725, Jul-Aug 2004.

[11]D. C. Hyun, M. Park, C. Park, B. Kim, Y. Xia, J. H. Hur, J. M. Kim, J. J. Park, and U. Jeong, "Ordered zigzag stripes of polymer gel/metal nanoparticle composites for highly stretchable conductive electrodes," Advanced Materials, vol. 23, pp. 2946-+, Jul 2011.

[12] T. Sekitani, Y. Noguchi, K. Hata, T. Fukushima, T. Aida, and T. Someya, "A rubberlike stretchable active matrix using elastic conductors," Science, vol. 321, pp. 1468-1472, Sep 2008.

[13] T. W. Tombler, C. W. Zhou, L. Alexseyev, J. Kong, H. J. Dai, L. Lei, C. S. Jayanthi, M. J. Tang, and S. Y. $\mathrm{Wu}$, "Reversible electromechanical characteristics of carbon nanotubes under local-probe manipulation," Nature, vol. 405, pp. 769-772, Jun 2000.

[14] L. B. Hu, W. Yuan, P. Brochu, G. Gruner, and Q. B. Pei, "Highly stretchable, conductive, and transparent nanotube thin films," Applied Physics Letters, vol. 94, Apr 2009.

[15] T. Yamada, Y. Hayamizu, Y. Yamamoto, Y. Yomogida, A. Izadi-Najafabadi, D. N. Futaba, and K. Hata, "A stretchable carbon nanotube strain sensor for human-motion detection," Nature Nanotechnology, vol. 6, pp. 296-301, May 2011.

\section{CONTACT}

* Y. Liu, E-mail: liu.yu2@husky.neu.edu and M. R. Dokmeci, Email: mdokmeci@rics.bwh.harvard.edu 